\title{
Deformable Image Registration With Inclusion of Auto-Detected Homologous Tissue Features
}

\author{
Y. Xie $^{1}$, L. Xing ${ }^{1}$, D. Paquin ${ }^{1}$, D. Levy ${ }^{1}$, T. Yang $^{2}$ \\ ${ }^{1}$ Stanford University, Stanford, CA, Northwestern Polytechnical University of China, Xi'an, China
}

Purpose/Objectives(s): Most image registration algorithms ignore the underlying tissue features but simply rely on the similarity of image intensity. As thus, a spatial accuracy better than $3 \sim 5 \mathrm{~mm}$ is hardly achievable using any of these techniques. The aim of this work is to develop a tissue feature-based deformable algorithm to substantially improve the performance of registration for various IGRT applications. The novelties of this work include: (1) auto-detection and quantitative characterization of homologous tissue features in the input images; and (2) seamlessly incorporation of the detected tissue feature information for accurate and robust registration.

Materials/Methods: The corresponding tissue features in the fixed and moving images are described by the information in the neighborhood of a point of interest. Quantitatively, the local information is characterized by using the Scale Invariance Feature Transformation (SIFT) method (the use of scalespace is to compare different images in the same scale, and is thus referred to as the SIFT), which includes scale-space extrema detection, control point localization, orientation assignment and control point descriptor. Another important feature of SIFT is the orientation histogram technique. In a 2D case, for example, the $8 \times 8$ neighborhood around a given point is defined as a volume. The volume is divided into four parts. In each part, the gradient magnitude of each pixel is calculated and sorted into 8 angle bins (the first bin is from 0 degree to 45 degree and so on). To further increase the precision of tissue feature association, a bi-directional mapping strategy is developed based on the intuitive fact that if a feature region in the fixed image is mapped correctly to the moving image, then it will necessarily be mapped back to the original feature region in the fixed image when we apply the inverse map to the corresponding feature region in the moving image. During the bi-directional calculation, the feature region is labeled as a matched region if the displacement vectors match. Otherwise, we consider it a mismatched region and delete it. The obtained homologous tissue features are treated as a priori knowledge in the subsequent deformable registration using thin-plate spline (TPS), or BSpline or finite element (FE) method.

Results: A theoretical framework of auto-determining homologous tissue features and incorporating the information into commonly used deformable registration algorithms have been established. Several experiments using both digital phantom and clinical thoracic cases demonstrate the accuracy and efficiency of the proposed method. For each case, the algorithm convergence is confirmed by starting the registration from a large number of initial transformation parameters. It is observed that the convergence behavior is substantially improved as a result of integrating a priori anatomic knowledge when compared with the conventional approaches. Markedly improved registration accuracy is observed as compared with the results obtained without incorporating tissue feature information for all cases.

Conclusions: Inclusion of prior anatomical knowledge is a key step in bringing the currently available deformable models to the next level. The proposed method provides a clinically practical solution to the complicated deformable registration problem and should be a valuable for IGRT applications. 